\title{
Toward a Quantitative Theory of Hofmeister Phenomena: from Quantum Effects to Thermodynamics
}

\author{
Travis P. Pollard and Thomas L. Beck \\ Department of Chemistry \\ University of Cincinnati \\ Cincinnati, OH 45221-0172 USA \\ thomas.beck@uc.edu
}

\begin{abstract}
Several recent developments have enhanced our understanding of specific ion hydration. These advances have included the Law of Matching Water Affinities and the realization that many-body dispersion forces and polarization can play important roles in ion specificity. Efforts have been made to partition the relevant ion free energies into their physically contributing parts in order to gain further insights into the driving forces. Yet a quantitative theory of ion specificity that links the necessary molecular-level treatment of the inner hydration shell with the many-body response of Lifshitz theory at longer range is still lacking. This review summarizes some steps toward quantitative models of specific ion hydration and discusses a possible path looking forward.
\end{abstract}

\section{Introduction}

One of the most influential ideas in our growing understanding of ion specificity has been the Law of Matching Water Affinities (LMWA) developed by Collins $[1]^{* *}$. This conceptual framework links two basic ideas: 1) the charge density of the ion is the important physical variable for specificity 2) the resulting water affinity of the ions determines the ion-ion interaction preferences following the general concept of "like prefers like." That is, in water, small, hard (kosmotropic) ions such as $\mathrm{Li}^{+}$ and $\mathrm{F}^{-}$prefer to interact closely with oppositely charged kosmotropes rather than with large, soft (chaotropic) ions such as $\mathrm{I}^{-}$or $\mathrm{Cs}^{+}$; the same holds for chaotropechaotrope pairs, while unlike pairs tend to dissociate in water.

While these electrostatics-based ideas are not a quantitative theory of specific ion hydration, they have played a crucial role in organizing the often dizzying array of 
data that appear for complex systems such as protein interactions in salt solution (Hofmeister and inverse Hofmeister series, etc.) [2, 3]. Collins has summarized this body of work in a recent review that suggests that continuum Debye-Hückel models are often not up to the task of explaining detailed ion-protein interactions $[4]^{*}$. In addition to the LMWA, Collins, Neilson, and Enderby [1 $]^{* *}$ made another key observation, namely that, on the local scale (due to the strong fields near ions in water), chemical effects such as charge transfer can play an important role in ion specificity.

Another major development has been the realization of the role of many-body dispersion forces in ion specificity led by Ninham and coworkers [5]**. This "top down" approach starting from Lifshitz theory (that involves quantum fluctuations over a broad frequency scale [6]) describes non-electrostatic effects included only approximately in simple force fields employed for molecular dynamics (MD) simulations. Some evidence will be discussed below that those simple classical MD models can in fact capture important features of ion specificity. Rather than diminishing the importance of dispersion forces, however, those simulation results, along with the recent continuum model results of Duignan et al. $[7,8,9,10,11,12,13]^{*}$ and quantum mechanical results presented below, suggest a middle-ground view may be the one that prevails. That is, we need to seriously consider all contributing factors, including cavity formation, electrostatics, dispersion, induction, charge transfer, and the realistic molecular structure of water in the first hydration shell, in order to make quantitative progress. The perhaps unsatisfying conclusion seems to be "all of the above."

On the experimental side, there is a vast body of information related to Hofmeister series in condensed matter chemistry and biology $[5,14,15]^{* *}$. The data is often confusing and seemingly contradictory, although there is no denying that the ion identity has a major impact on both microscopic structure and macroscopic thermodynamics. A noteworthy point was made by Evens and Niedz in 2008 [16] ${ }^{* *}$. They showed that most often Hofmeister-related observations are made in a highdimensional space of variables - what is typically measured is a cut through that high-dimensional space, where in reality there are significant influences of several composition variables rather than just one. That observation doesn't solve the problem it raises, but it does suggest caution in interpreting experimental data.

With these theoretical and experimental complexities, a natural impulse might then be to throw up our hands in defeat. Still, there is no doubt that progress has been made over the last two decades, at least in elucidating the important factors that should now be addressed with a more quantitative theory. This review attempts to cover some of that progress. There has been extensive work employing classical 
computer simulations to study interesting mesoscopic specific ion effects and relating them to measured thermodynamic properties $[17,18,19]$. Here we will focus on the small and the large: detailed quantum mechanics for the inner-shell interactions, and the computation of macroscopic thermodynamic properties that can be related to precise measurements. We argue that the former is necessary for the latter.

\section{Goals for a quantitative theory}

We first outline a series of what we view as key goals or steps on the path to a more quantitative theory of specific ion effects. In the following sections we assess the current status of work related to each step. As stated in Ref. [8]*, "The only certain thing is that any progress has to begin with a quantitative theory of the solvation free energies of the ions." But already this raises follow-up questions, namely what are the single-ion hydration free energies and how do we get them from experiment?

The quest for establishing single-ion solvation quantities has been pursued for well over 100 years without a clear resolution $[20,21,22,23,24]^{* *}$. Thus the first goal is to determine, quantitatively, a single-ion free energy scale, along with enthalpy and entropy scales. Without such an anchor, quantitative results are not possible. As recognized repeatedly in the literature, in order to define single-ion quantities, we must deal with interfacial potential effects $[25,20,26]^{*}$. Establishing a defensible value for the effective surface potential of water is thus a major objective, as is clarifying its meaning. While the interfacial potential has no impact on the bulk properties of ionic solutions, it can have physical consequences near interfaces by affecting ion distributions and even the acid-base chemistry of water itself $[27,28$, $29,23]^{* *}$. Thus the interfacial potential is of more than just academic interest.

Second, it will be helpful to continue to relate computed properties to other accessible measured structural and thermodynamic quantities (at increasing levels of resolution). These quantities include local structural information obtained from probes such as XAFS [30]*, XPS [31], nonlinear spectroscopies [32], and NMR [33]. Other thermodynamic properties include surface tension and surface potential increments (with changing concentration) $[34,18]$ and bulk osmotic and activity coefficients $[18,35]^{*}$. In addition, impacts of detailed quantum mechanical effects (such as charge transfer) on ion transport should be investigated in more detail [36].

Third, we should further assess what level of theory is necessary to obtain acceptable agreement with the data obtained in the first step. Part of this analysis involves spatial partitioning of the free energies to determine which regions require high-accuracy quantum treatments and which can be handled with simplified force field models or even continuum methods [37, 38, 39, 40, 41, 42 $]^{*}$. Temperature derivative quantities $[42]^{*}$ are more difficult to model than the free energies themselves, so 
there should be focus on those quantities, especially the entropies (since they are less affected by interfacial potential effects, see below).

Fourth, starting from accurate quantum chemistry, we need to gain a better understanding of the contributing parts of the ion-solvent interaction energies [12]. This can be accomplished using quantum chemical perturbation theory (symmetry adapted perturbation theory [43, 44], SAPT, see below). It should be noted there is no unique partitioning of the energies (either in quantum chemistry or in statistical mechanics), but that does not mean no insight can be gained. The factors to examine include exchange, electrostatics, dispersion, induction, and charge transfer (which is often argued to be included in the induction energy [43, 45]). Also, examination of electronic charge redistributions [46, 47] upon forming ion-water clusters is helpful for seeing the complexity of ion hydration and to interrogate simple classical models.

Finally, along with the single-ion quantities, we should dig deeper into the ionion interaction issue in water as solvent $[5]^{* *}$. This involves computing the potential of mean force (PMF) between the ions, which can then be input to approximate theories for ion-pair activities and osmotic coefficients for direct comparison with experimental data. Extensive classical simulations $[18,48]$ and continuum dispersion modeling [13] have been directed at these PMFs and their ion specificity, but it is clear that classical models can struggle in reproducing the close-contact structure and energetics (where the PMFs vary the most) $[49,50]^{*}$. Thus extending the previous work to more accurate quantum models is desirable.

We will go through these goals in turn and discuss the current status of each. The review is necessarily incomplete, but we attempt to give a representative overview of each topic.

\section{Single-ion thermodynamic quantities and interfacial potentials}

Prominent individuals (Gibbs and Guggenheim [51, 52, 53]) have stated that potential shifts experienced by single ions moving across interfaces are not thermodynamically measurable: "The electric potential difference between two points in different media can never be measured and has not yet been defined in terms of physical realities. It is therefore a conception which has no physical significance" $[52]$.

Nevertheless, the real electrochemical hydration free energy for a single ion (advocated by Guggenheim as the only physically realizable single-ion free energy, not its "chemical and potential" parts) is often expressed $[54,55,26]^{* *}$ as

$$
\mu_{\text {real }}^{e x}=\mu_{b u l k}^{e x}+q \phi_{n p}=\mu_{i n t r}^{e x}+q \phi s p
$$


where the left side is the (real) free energy to transfer an ion from the vapor phase deep into the liquid, $\mu_{b u l k}^{e x}$ is the free energy change without interfacial potential effects, and $\mu_{i n t r}^{e x}$ includes all interactions with the solvent except for an electrostatic contribution (the surface potential) from a distant water surface (see Figure 1). The surface potential $\phi_{s p}$ (obtained by direct integration across the interface [54] ${ }^{* *}$ ) is of magnitude $4 \mathrm{~V}[56,57]$ or roughly $90 \mathrm{kcal} / \mathrm{mol}$-e for water represented with a realistic quantum mechanical charge distribution - a large cancellation between the liquidvapor surface and the ion-water boundary due to a quadrupole effect results in a net potential an order of magnitude smaller $[58,57,26,59]^{*}$. The difference between classical and quantum models is striking (roughly values of order $-10 \mathrm{kcal} / \mathrm{mol}-\mathrm{e}$ [60] and $+90 \mathrm{kcal} / \mathrm{mol}-\mathrm{e}$, respectively $[56,57])$. The local potential in quantum mechanical water is of comparable magnitude to the surface potential, implying the intrinsic quantities are also highly sensitive to the model.

The relation between the potentials is $\phi_{n p}=\phi_{l p}+\phi_{s p}$ where $\phi_{n p}$ is the potential shift on passing from vacuum into the liquid and then into a mesoscopic-sized cavity in water, $\phi_{l p}$ is the potential shift crossing the ion/water boundary (local potential), and $\phi_{s p}$ is the potential crossing the liquid-vapor surface (see Figure 1). Any division into chemical and electrostatic parts as done in the two forms given above (Eq. 1) involves an artificial partitioning of the Coulomb potential and is thus not directly measurable (but is computable). This is the origin of Guggenheim's statement.

In a rigorous sense, Guggenheim's comment is certainly correct, but this does not mean we should completely avoid the question: Is there an average potential an ion "feels" near the liquid-vapor interface of water? If such a potential exists (as we would expect for a molecular liquid with a charge asymmetry and hydrogen bonding possibilities, etc.), it should be a property of the self-organized structure of water itself and thus should exert its influence only through the ion charge (as indicated in Eq. 1). A consistent length scale has been discovered for monovalent ion hydration that suggests a physical basis for defining a net potential $[61]^{*}$. Namely, the net potential is the difference between the real hydration free energy and minus the work necessary to push the nearby water molecules out to a length scale of roughly 6 $\AA$; the cavity formation calculation around the ion produces the bulk hydration free energy.

Refs. $[62,23]^{* *}$ have shown, on the other hand, that the real single-ion free energy $\mu_{\text {real }}^{e x}$ (that includes a contribution from the water surface potential) can be obtained directly from cluster and bulk hydration experiments without any extrathermodynamic assumptions and/or the use of computational models. Using this observation, re-evaluations of the current standard for the thermodynamics of proton hydration [63] were made. Once the values are established for a single ion, 
values for all other ions are obtainable from accurate bulk thermodynamic data. Refs. $[22,23]^{* *}$ estimated these proton values (for passing from a $1 \mathrm{M}$ ideal gas to a $1 \mathrm{M}$ ideal solution) as $-264.2 \mathrm{kcal} / \mathrm{mol}$ for the free energy, $-271.6 \mathrm{kcal} / \mathrm{mol}$ for the enthalpy, and $-24.5 \mathrm{cal} / \mathrm{mol}-\mathrm{K}$ for the entropy.

In Ref. [22]*, the cluster pair approximation (CPA) for the proton quantities [63] was re-examined theoretically and computationally from the standpoint of the quasichemical theory (QCT) of ion hydration. The CPA is widely accepted as the "gold standard" for single-ion free energies. The study shows that the free energies and enthalpies obtained from the CPA approach are the real quantities discussed above, but the CPA contains an extra-thermodynamic assumption that may affect the resulting values. By examining clusters of increasing size in polarizable (AMOEBA) simulation models of the $\mathrm{NaF}$ (kosmotropic) and RbI (chaotropic) ion pairs, the real hydration free energy, enthalpy, and entropy of the proton were calculated, exhibiting remarkable agreement with the values determined directly from the experimental data discussed above. Shifts in the values with increasing cluster size were subsequently confirmed for the $\mathrm{NaF}$ pair using MP2-level electronic structure calculations $[23]^{* *}$. A similar analysis was carried out in Refs. [21]* and [24] $]^{* *}$ that focused on the evolution of the surface potential $\phi_{s p}$ with cluster size.

The estimated hydration entropy for the proton $(-24.5 \mathrm{cal} / \mathrm{mol}-\mathrm{K})$ is very close to that predicted long ago by Conway [25]. The statistical mechanical formula (assuming low pressure) for the single-ion entropy is [26]

$$
s^{e x}=U_{S R} / T-k \ln \langle\exp [(\varepsilon-\langle\varepsilon\rangle) / k T]\rangle \approx U_{S R} / T-\left\langle\delta \varepsilon^{2}\right\rangle / k T^{2}
$$

where $U_{S R}$ is the change in the average solvent total energy upon adding the ion (solvent reorganization energy), $\varepsilon$ is the interaction energy of the ion with the solvent (not necessarily pairwise decomposable), $\delta \varepsilon$ is the difference from the mean value, and the averaging indicated by the brackets is conducted with the ion present in the solution. The last approximate (Gaussian) form shows that the entropy involves a significant cancellation between a typically positive solvent reorganization energy and a negative fluctuation term. This balance is the reason ion hydration entropies are of such small magnitude $[64,42]^{*}$, comparable to rare gas pairs (see Fig. 2 below). It is clear from this formula that the entropy depends only weakly if at all on the net potential since $\phi_{n p}$ is not present in $U_{S R}$ and should cancel in $\delta \varepsilon$. Thus bulk and real estimates of the entropy should be close to each other, as is observed.

Ref. [21 $]^{*}$ made a similar analysis of the size dependence of the predicted free energies using a classical SPC/E point charge model of water. The surface potential $\phi_{s p}$ was examined as a function of cluster size and was found to approach an asymptotic value only for large cluster sizes. The study confirms the need for shifts in the 
previous CPA values, but the resulting proton values do not agree with the experimental results for the real quantities discussed above. In particular, the entropy is of very small magnitude (similar to that for the chaotropic $\mathrm{I}^{-}$ion). The deviation is likely due to the model employed. Also, as discussed above, the individual $\phi_{l p}$ and $\phi_{s p}$ display significant model dependence with quantum and classical results differing by around $100 \mathrm{kcal} / \mathrm{mol}$-e; there exists large differences between water models too (such as SPC/E vs. TIP5P water) [59]*. All of these effects are tied to quadupolar contributions discussed in several papers $[65,59,66]^{*}$.

By comparison with bulk values obtained from the Marcus (net potential free $\left.[22]^{*}\right)$ tables, a surface potential value of approximately $-0.4 \mathrm{~V}$ was obtained. Ref. [24]** discusses a valid concern that the Marcus data does not add properly to the known bulk pair data (due to "the inconsistent use of gas and liquid phase formation Gibbs free energies.") We note, however, that for the two pairs examined in Ref. [22]* (NaF and $\mathrm{RbI}$ ) the errors are of magnitude $1.4 \mathrm{kcal} / \mathrm{mol}$ (in comparison with the data in [63]); if the errors were partitioned evenly between the ions and the maximum possible deviation assumed, this would alter our surface potential estimate by $10 \%$ or less. Finally, two independent lines of theoretical work $[67,68]^{*}$ (that contain no surface potential effects) produce exactly the same proton free energy as the one from the Marcus table, supporting the validity of identifying it with the bulk value. Note there are major subtleties related to interfacial potentials in bulk samples: as soon as multiple ions are involved the net potential becomes ion specific (these issues are discussed in Ref. [23] ${ }^{* *}$ ). Still the solvent and ion contributions can to a large extent be considered separately; here we are discussing the solvent contribution.

As discussed above, there is no direct measurement of the electrochemical surface potential (or net potential) of water. There are two indications that the value is close to $-0.4 \mathrm{~V}$, however. One is a study of ion distributions near a hydrophobic ion pair (TATB) near the water/dichloro-ethane surface $[27]^{*}$ (whose implications are discussed in Ref. [23]**). The second is the isoelectric point of bubbles (and oil droplets) in water [28] and indications from electrospray mass spectrometric measurements [29]. Both of these experiments suggest the surface is neutralized at a bulk $\mathrm{pH}$ of $3-4$. A simple electrostatic analysis using the $-0.4 \mathrm{~V}$ net potential value and the fact that only about half the potential drop occurs inside the region of the liquid accessible to ions produces a $\mathrm{pK}_{a}$ shift right in this range. A direct computation of the net potential in recent $a b$ initio simulations [59] produced a significantly smaller magnitude net potential. Possible reasons for the discrepancy are discussed in Refs. $[22,23]^{* *}$.

In an impressive series of recent papers, Duignan et al. have developed a continuum multipolar theory of dispersion interactions involved in ion hydration. The 
general method was developed in [7] and then applied to studies of single-ion hydration free energies [8]*, partial molar volumes and entropies [9], ions at the air-water interface [10,11] (see also [69]), and ion-ion interactions leading to predictions of activity and osmotic coefficients $[12,13]^{*}$. The work has led to good agreement with experiment in terms of general trends of ion specificity, with some difficulty in reproducing quantities such as hydration entropies. It is our opinion that this work expresses the state-of-the-art in continuum modeling of ion hydration and shows the contributing role that dispersion plays in ion specificity. We will argue below that additional features are also necessary for quantitative theory, namely a molecular-level treatment of the first hydration shell.

It is clear that progress has been made over the last several years in quantifying the meaning of single-ion thermodynamic quantities that appear across many areas of chemistry. The hydration entropy is discussed further below in relation to bulk thermodynamics for ion pairs and free energy partitioning models.

\section{Other experimental thermodynamic and structural quantities}

Many theory and simulation studies have related calculated results to bulk thermodynamic measurements beyond the single-ion quantities discussed in the previous section. These studies have included surface tension $[70,71,18]$ and surface potential $[18,70]$ increments with increasing ion concentration, basic ion pair thermodynamic hydration quantities (with no ambituities from surface potential effects) [72], ion entropies $[42]^{*}$ that are related to the phase behavior of polymer solutions [73], and specific ion effects on protein structure and protein-protein interactions (both standard and reverse Hofmeister series depending on the protein charge) [17, 2, 3, 74, 75]. Theory and experiment for ion-ion interactions relevant to osmotic coefficients, ion activities [76], and the occurrence of ion pairing will be discussed below.

Since computations of surface tension and surface potential increments require large systems in order to avoid appreciable system size effects, most studies have involved classical simulations with simple point charge models. Netz and Horinek [19] reviewed the development of optimized ion-water force fields based on a combination of Gibbs free energy, entropy, and structural experimental data. Several parameter sets were developed and those force fields were tested vs. experimental surface tension increments. Generally, the results are in decent agreement with experiment, but nontrivial sensitivity of the results to details of the parameters was observed. The variation of the surface potential with the ion identity was also analyzed [18] (the data in Fig. 17 of Ref. [18] appear to be oriented with the opposite sign from the more standard one passing from the vapor into the water phase). Those particular 
simulation data were not compared with experiment, but for the water/hydrophobic interface the results appear consistent with previous experiments summarized in Refs. [34] and [70]. Comparisons to experimental surface potential increment data were made in Ref. [70].

On larger scales, classical simulation has been employed to examine specific ion effects on protein-protein interactions [17] and protein structure [2]. In Ref. [17] the protein-protein potential of mean force (PMF) was computed for lysozyme at low and high $\mathrm{pH}$ conditions (with an accompanying change of the charge of the protein). A continuum solvent was assumed due to the very large size of the system. In agreement with experiment, the simulations predicted salting out of negatively charged proteins that follows the direct Hofmeister series and the reverse for positively charged proteins. The PMF profile produces the second virial coefficient for the protein solution. Similar agreement with experiment was observed in extensive models of the interactions of ion pairs with hydrophobic and hydrophilic surfaces [77]. The results help to understand the reversal of the Hofmesiter series going from hydrophobic to hydrophilic surfaces.

The thermodynamics of adsorption of the chaotropic thiocyanate anion to the water surface was recently measured using the temperature dependence of resonant UV second harmonic generation spectroscopy [32]. The data were modeled using molecular dynamics simulations and conclusions were drawn regarding a role for water-water interactions and the suppression of capillary waves by ions at the surface (leading to negative adsorption entropies). There has been some disagreement in the literature concerning the role of water-water interactions in the driving forces since the same term appears with opposite sign in the enthalpy and entropy [26]. Several other experiments have probed the occurrence of ions at the water liquidvapor surface, reviewed in Refs. [78] and [19], and continuum models have yielded good agreement with experimental surface excesses [69, 10].

An interesting thermodynamic example is provided by the study of ion entropies in Ref. [42]* There has been a long-standing mystery related to the near equality of the hydration entropies for the $\mathrm{KCl}$ pair and $2 \mathrm{Ar}$ atoms. A compensation between solvent and interaction energy fluctuation contributions to the entropy was discussed above. Fig. 2 shows a comparison of data for ion pair entropies with the corresponding rare gas pairs. The data show an anti-correlation with $\mathrm{KCl}$ appearing near the crossing point. These results make sense since the entropy for nonpolar particles becomes more negative with increasing size, with the opposite behavior for ions. Also note the excellent agreement of the computed and experimental ion pair results (except for the problematic $\mathrm{F}^{-}$ion, see below). Pair free energies of hydration computed with the AMOEBA model also agree very well with experiment [72]. 
Finally, coupled theory and experimental work has appeared over the last several years aimed at studying in detail the local structure around ions in water. These studies have included XAFS analysis of the very different solvation structure around the $\mathrm{I}^{-}[30]^{*}$ and iodate [79]* ions, and NMR studies that examine ion adsorption near surfactant charged surfaces [33]. The XAFS results and related simulations show clearly the importance of detailed quantum effects in the inner shell not captured by polarizable classical models. We view these and studies like them as essential in establishing benchmarks for the development of better models.

\section{Theory level and statistical mechanical partitioning}

A picture that emerges from the studies discussed above is that well-parametrized classical models can capture crucial aspects of specific ion hydration, including high resolution single-ion thermodynamic quantites. One success story is the AMOEBA force field that is mainly based on high-level quantum chemical calculations [72]. On the other hand, there appears to be high sensitivity of the thermodynamic results to fine-tuned details of the models. An example is the frequently observed over-polarization of classical models [80] in comparison with more realistic quantum models $[81,82]^{*}$. That effect can be corrected by variation of a damping parameter employed in the self-consistent polarization calculation $[22]^{*}$, but it does point out the possible limitations of the models.

For these reasons, well-optimized classical point charge models [19] have been shown to perform as well if not better than polarized models in studies of $\mathrm{I}^{-}$near the water liquid-vapor surface compared with ab initio simulations [82]*. Namely, the polarizable classical models have shown a deeper free energy minimum [80] for the ion at the surface than for the PMF profile modeled with ab initio simulation. In addition, we re-iterate the importance of proper inclusion/exclusion of interfacial potential effects when developing classical models based on experimental free energies. Neglect of this issue can lead to errors of magnitude $10 \mathrm{kcal} / \mathrm{mol}$ in the models, which are often larger than the specific ion effects we seek to study.

Besides these more technical issues, classical simulations often leave unanswered basic physical questions such as: 1) Which parts of the interactions dominate (cavity formation, dispersion, electrostatics, induction, etc.)? 2) What are the range of length scales that are involved in the interactions? Recent work has helped to provide a direction towards answering some of these questions.

The first theoretical approach is the quasi-chemical theory (QCT) [67, 37, 83, 38, $84]^{*}$. That theory employs a spatial partitioning enacted by manipulations involving hard particles during the process of inserting the solute into water. Three free energy 
terms result: 1) inner-shell 2) packing and 3) outer-shell (see Fig. 1). In turn these free energies correspond to: 1) minus the free energy to push nearby waters out to the hard particle radius 2) the free energy to create a cavity the size of the hard particle and 3) the free energy to insert the solute particle into the cavity created by the hard particle. Several studies have examined the behavior of each term as a function of cavity size (see Ref. [61]* as a recent example related to ion specificity), and the inner-shell term can be re-expressed in a chemical equilibrium form that allows for accurate quantum calculations [39]*. Besides the theoretical insights the QCT has generated, the theory has been shown to produce accurate hydration free energies for the alkali cation series $[37,39,85]^{*}$.

An alternative view starts from the Local Molecular Field Theory (LMFT) of Weeks and coworkers [86]. The LMFT has proved helpful for understanding structure and driving forces in molecular liquids and at interfaces. The basis of the LMFT for polar liquids like water is an Ewald-like partitioning of the Coulomb potential into near-field and far-field parts. That idea was employed in a new approach for partitioning the free energies [40] which was then utilized in studies of ions near the water surface [41], ion hydration entropies [42]*, ion thermodynamics in non-aqueous solvents [87], and ion-ion interactions related to the Law of Matching Water Affinities (Shi and Beck, in preparation).

As an example, Fig. 3 shows the near and far field electrostatic parts of the single-ion entropies computed in Ref. [42]* as a function of the inverse of the ion crystal radius. This plot yields insights into several physical aspects of specific ion hydration as well as explains the origin of the entropy plot used by Collins, Neilson, and Enderby $[1]^{* *}$ to show a clear indication of kosmotopric and chaotropic behavior. For this plot, the free energies were spatially partitioned into near-field and far-field components. What is clear is that it is the local electrostatic contribution that is the predominant source of ion specificity (with some variation from the van der Waals component, see Table 2 of Ref. [42]*). The far-field electrostatic part of the free energy produces only a small nearly ion independent negative value.

Thus it appears ion specificity is remarkably local (at least for monovalent ions). Also, it was noted in Ref. [38] that simple dielectric models can underestimate the magnitude of ion entropies by up to an order of magnitude; clearly that observation is tied to molecular-level behavior in the first shell. A similar conclusion was reached in a study of IR and Ramam spectra of H-bonds around ions in water [88] and in a QCT study of ion hydration [89].

The QCT and the LMFT partitioning have produced similar physical results, namely that molecular-level quantum mechanical accuracy is required for the nearby interactions of the ion with water. But, due to screening and resulting significantly 
reduced field intensity, the more distant interactions are well-modeled as a Gaussian process that is equivalent to a dielectric continuum model. These ideas have been known qualitatively for a long time, but it has been helpful to see computationally that outside of the first hydration shell, the interactions are truly Gaussian distributed [40]. This is the reason the Born and related continuum models have persisted. These conclusions appear robust in bulk water at low ion concentration, but Collins has pointed out several cases where the continuum-based Debye-Hückel model for ion-ion interactions fails regarding ion-protein interactions $[4]^{*}$.

\section{Quantum structure and energetic partitioning}

The fluctuating fields near ions in water can be enormous (of magnitude $1 \mathrm{~V} / \AA$ ) $[90]^{*}$. It is therefore not surprising that the ion charge cloud can become substantially distorted, especially for the anions. While classical models can to some extent capture key physical aspects of ionic solutions such as the ion size, electrostatic interactions, and approximations to ion polarization and charge transfer, the QCT results discussed above suggest that an accurate quantum mechanical treatment of the inner-shell is necessary for high resolution thermodynamic results. In addition, high-level quantum chemistry can produce insights into the contributing physical parts of the interactions through perturbation theory (such as SAPT) [43, 91]. In this section we summarize some previously unpublished quantum mechanical results related to ions in water clusters (see supplementary materials for details). A previous study examined ion specificity of dispersion interactions [12], but here we extend the results to all of the relevant interactions. We also explore charge transfer effects that have been studied previously $[92,81]$. There is experimental evidence of charge transfer in ion hydration [93].

The main conclusions of our work are as follows.

- Significant ion specificity is observed for each of the interaction energy components using SAPT. The micro-solvated ion/water interaction energies increase non-linearly as waters are added. See Table 1.

- The Quantum Theory of Atoms in Molecules [46] quantities calculated at the intermolecular bond critical point for ion/water dimers hint that the $\mathrm{F}^{-}$/water hydrogen bond is uniquely strong among the halide/water series. This is also evidenced by significant induction and dispersion contributions to the interaction energy. Electrostatics dominates all the interactions examined, however.

- Cations are found to accommodate up to 80 millielectrons of charge from nearby 


\begin{tabular}{|c|c|c|c|c|c|c|c|}
\hline Cluster & Elst & Ind & Disp & Exch & Exch Err & $\mathrm{E}^{\mathrm{PT}}$ & $\mathrm{E}^{\mathrm{CP}}$ \\
\hline \multicolumn{8}{|c|}{$\mathbf{X}^{ \pm}\left(\mathbf{H}_{2} \mathrm{O}\right)$} \\
\hline $\mathrm{Li}^{+} \mathrm{C}_{2 \mathrm{v}}$ & -32.90 & -13.52 & -0.68 & 12.55 & 1.00 & -34.56 & -33.46 \\
\hline $\mathrm{Na}^{+} \mathrm{C}_{2 \mathrm{v}}$ & -24.95 & -6.39 & -0.43 & 8.43 & 1.00 & -23.33 & -22.53 \\
\hline $\mathrm{K}^{+} \mathrm{C}_{2 \mathrm{v}}$ & -19.73 & -4.78 & -1.73 & 8.73 & 1.01 & -17.51 & -16.63 \\
\hline $\mathrm{F}^{-} \mathrm{C}_{1}$ & -44.50 & -29.26 & -7.80 & 49.33 & 1.03 & -32.23 & -29.35 \\
\hline $\mathrm{Cl}^{-} \mathrm{C}_{1}$ & -19.01 & -7.57 & -4.42 & 15.41 & 1.01 & -15.58 & -14.24 \\
\hline $\mathrm{Br}^{-} \mathrm{C}_{1}$ & -16.35 & -6.03 & -4.15 & 13.13 & 1.01 & -13.39 & -12.18 \\
\hline
\end{tabular}

Table 1: SAPT2+3(CCD) energy decomposition for ion-water dimers: electrostatics (Elst), induction (Ind), dispersion (Disp), exchange (Exch), and total interaction energy $\left(\mathrm{E}^{\mathrm{PT}}\right)$ in the dimercentered aug-cc-pVTZ basis set. All energies expressed in kcal/mol. Exch Err is a value quantifying the degree of error in the single-exchange approximation which affects the accuracy of exchangecoupled higher order induction terms. A value greater than unity indicates an overestimation of the induction energy and vice versa. This quantity represents the chief source of error in the difference between $\mathrm{E}^{\mathrm{PT}}$ and the conventional counterpoise-corrected interaction energy, $\mathrm{E}^{\mathrm{CP}}$.

waters while anions are found to distribute up to 170 millielectrons to the solvent - neither increase linearly as waters are added.

- Density difference maps of the anion complexes reveal the scope and sheer complexity of the charge redistribution between ions and nearby waters. See Fig. 4. See also Ref. [94] for related analysis of the water molecule.

- An alternative method that displays the charge redistribution in small complexes [47] is helpful to visualize the complicated charge flow in the inner shell. Again, anions display larger extents of charge rearrangement, espcially for the $\mathrm{F}^{-}$ion. See Fig. 5. All three of these last observations regarding charge redistributions display strong ion specificity.

It is clear from these results and others [95] that the local fields are intense and the charge rearrangements are extensive and complicated. There have been recent efforts to develop classical models that include both polarization and charge transfer [96]. These models appear to be promising in producing good agreement with experiment while maintaining the computational efficiency needed for large-scale simulations. Application of these models to ion association will be discussed below [35]* We also note recent application of these ideas to ion transport [36]. 


\section{PMFs and ion-ion interactions}

Along with the single-ion entropies discussed above, another hallmark indicator of ion specificity (and the LMWA) is the volcano plot assembled by Collins, Neilson, and Enderby $[1]^{* *}$. That plot shows the heat of solution (from the crystal) for ion pairs on the y axis vs. the difference of the ion pair hydration enthalpies (from vapor into liquid) on the $\mathrm{x}$ axis. Like-like pairs (chaotrope-chaotrope and kosmotropekosmotrope) generally display positive heats of solution while unlike pairs show the opposite behavior. Another plot that displays volcano behavior is one that shows the osmotic coefficient on the y axis and the difference of solvation free energies for the pair on the $\mathrm{x}$ axis $[13,35]^{*}$. Recent computational studies that employ polarizable and charge transfer force fields have successfully modeled the osmotic coefficients vs. total ion concentration $[35]^{*}$.

The basic theory for computing the osmotic coefficients is given in Ref. [13] ${ }^{*}$. The computation involves a second virial coefficient term that requires the ion-ion PMF and another term that depends only on the ion valency (long-ranged interactions that can often be modeled at the Debye-Hückel level at least in homogeneous solutions). Ref. [13]* examined the osmotic coefficients vs. the anion-cation difference of solvation free energies using a continuum model; the same general volcano plot was observed in the calculations compared with experiment, but the theoretical results were shifted relatively uniformly on the y-axis. These results again implicate the importance of dispersion forces, but also point to the need for the inclusion of other physical effects. At any rate, the central object of interest is an accurate ion-ion PMF for each pair.

Several groups have performed extensive classical simulations of the ion pair PMFs in water. Fennell et al. [48] examined a range of ion pairs using several force fields for the ions and water. The results were generally consistent with the LMWA, and for the OPLS parameter set computed association constants agreed relatively well with experiment. As for the surface tension increments above, the PMFs display strong sensitivity to the ion and water models. Some of the force field parameters were subsequently corrected [48] which led to better agreement with experiment. Ion pair PMFs were also computed in Ref. [18] using the point charge force fields obtained by matching experimental free energies, entropies and structural data. The resulting osmotic coefficients vs. ion concentration agree quite well with experiment. Ref. [97] observed results in decent agreement with the LMWA except for ion sizes near the size of the water molecule where deviations were observed.

Recently, efforts have been directed at ab initio simulation studies of ion-ion PMFs to test the validity of the classical models. Here we mention two of these studies that serve to illustrate the strong dependence of the PMFs on the classical force field in 
relation to the quantum mechanical results $[49,50]^{*}$. A study discussing an LMFT partitioning of the ion-ion PMFs will appear shortly (Shi and Beck, in preparation); as for the single-ion quantities, the partitioning aids in understanding the underlying driving forces for the observed PMF features.

\section{Conclusions}

The above discussion gives a glimpse of recent efforts to move theoretical and modeling research on Hofmeister effects in a quantitative direction. Progress has been made in defining, understanding, and modeling single-ion thermodyanmic quantities. We view this as a necessary first step along the path to higher resolution results. Studies of other thermodyanmic and structural quantities have allowed for robust testing of frequently employed classical and quantum models. Spatial partitioning of free energies and temperature derivative quantities has led to a better understanding of the physical basis of the near locality of ion specificity. Detailed quantum studies, while computationally expensive, can serve as benchmarks for determining the magnitudes of the various factors that contribute to ion specificity.

Recurrent themes in the research discussed here are 1) Classical models can capture important features of ion specificity when the models are robustly parametrized vs. experimental quantities or high-level quantum results. 2) Yet for higher-resolution theory aimed at quantitative results, it is clear the quantum mechanical theories and calculations are required. There is no avoiding the physical fact of the intense fields in and around ions in water, and the impact of those fields on the electron distributions are complex.

In the Abstract above, it was stated that a theory that links molecular-level reality in the inner hydration shell to the continuum Lifshitz theory at longer range doesn't exist. The quasichemical theory comes close to this goal by providing a statistical framework for parsing the physical effects into quantum results at short range and continuum-level electrostatic models at long range. A step beyond would be to include more than just the electrostatic effects at long range by adding dispersion interactions of the ion-water cluster (or "dressed" ion) using Lifshitz theory.

We also suggest that in order to move forward in determining interfacial potential effects, it appears necessary from the beginning to perform calculations that include the realistic quantum mechanical charge distributions of the ions and water molecules. This is due to the clear model dependence in classical models in which the charge distributions are decidedly unphysical inside the molecules. Applying the ideas discussed here to important non-aqueous solvents is another direction for further exploration [87]. 


\section{Acknowledgements}

This research was supported by the National Science Foundation under grants CHE-1011746 and CHE-1266105. We thank the Ohio Supercomputer Center for a generous grant of computer time. We also thank Lawrence Pratt, Chris Mundy, Shawn Kathmann, Greg Schenter, Tim Duignan, Drew Parsons, Barry Ninham, and Pierandrea Lo Nostro for many helpful conversations.

\section{References}

[1] ${ }^{* *}$ Kim D. Collins, George W. Neilson, and John E. Enderby. Ions in water: Characterizing the forces that control chemical processes and biological structure. Biophysical Chemistry, 128(2-3):95-104, July 2007. Insightful review that discusses ion specificity, the Law of Matching Water Affinities, and the importance of charge transfer.

[2] Kelvin B Rembert, Jana Paterova, Jan Heyda, Christian Hilty, Pavel Jungwirth, and Paul S Cremer. Molecular mechanisms of ion-specific effects on proteins. Journal of the American Chemical Society, 134(24):10039-10046, 2012.

[3] Jana Paterova, Kelvin B Rembert, Jan Heyda, Yadagiri Kurra, Halil I Okur, Wenshe R Liu, Christian Hilty, Paul S Cremer, and Pavel Jungwirth. Reversal of the Hofmeister series: Specific ion effects on peptides. The Journal of Physical Chemistry B, 117(27):8150-8158, 2013.

[4] *Kim D Collins. Why continuum electrostatics theories cannot explain biological structure, polyelectrolytes or ionic strength effects in ion-protein interactions. Biophysical chemistry, 167:43-59, 2012.

[5] **Barry W. Ninham and Pierandrea Lo Nostro. Molecular Forces and Self Assembly in Colloid, Nano Sciences and Biology. Cambridge, Cambridge, 2010. Stimulating book that gives a broad overview of Hofmeister effects and the important role of dispersion forces.

[6] V. Adrian Parsegian. Van der Waals Forces: A Handbook for Biologists, Chemists, Engineers, and Physicists. Cambridge, Cambridge, 2006.

[7] Timothy T Duignan, Drew F Parsons, and Barry W Ninham. A continuum solvent model of the multipolar dispersion solvation energy. The Journal of Physical Chemistry B, 117(32):9412-9420, 2013. 
[8] *Timothy T Duignan, Drew F Parsons, and Barry W Ninham. A continuum model of solvation energies including electrostatic, dispersion, and cavity contributions. The Journal of Physical Chemistry B, 117(32):9421-9429, 2013.

[9] Timothy T Duignan, Drew F Parsons, and Barry W Ninham. A continuum solvent model of the partial molar volumes and entropies of ionic solvation. The Journal of Physical Chemistry B, 118(11):3122-3132, 2014.

[10] Timothy T Duignan, Drew F Parsons, and Barry W Ninham. Ion interactions with the air-water interface using a continuum solvent model. The Journal of Physical Chemistry B, 118(29):8700-8710, 2014.

[11] Timothy T Duignan, Drew F Parsons, and Barry W Ninham. Hydronium and hydroxide at the air-water interface with a continuum solvent model. Chemical Physics Letters, 635:1-12, 2015.

[12] Timothy T Duignan, Drew F Parsons, and Barry W Ninham. Collins's rule, hofmeister effects and ionic dispersion interactions. Chemical Physics Letters, 608:55-59, 2014.

[13] *Timothy T Duignan, Drew F Parsons, and Barry W Ninham. A continuum solvent model of ion-ion interactions in water. Physical Chemistry Chemical Physics, 16(40):22014-22027, 2014.

[14] *P. Lo Nostro and B. W. Ninham. Hofmeister phenomena: an update on ion specificity in biology. Chemical reviews, 112(4):2286-2322, 2012.

$[15]{ }^{*}$ V. Craig and C. Henry. Specific ion effects at the air-water interface: Experimental studies. In W. Kunz, editor, Specific Ion Effects. World Scientific, New York, 2010.

[16] ${ }^{* *}$ Terence J Evens and Randall P Niedz. Are Hofmeister series relevant to modern ion-specific effects research? Scholarly Research Exchange, 2008, 2008. http://dx.doi.org/10.3814/2008/818461.

[17] M. Lund, J. Heyda, and P. Jungwirth. Ion binding to biomolecules. In W. Kunz, editor, Specific Ion Effects. World Scientific, New York, 2010.

[18] J. Dzubiella, M. Fyta, D. Horinek, I. Kalchev, R. Netz, and N. Schwierz. Ionspecificity: From solvation thermodynamics to molecular simulations and back. In W. Kunz, editor, Specific Ion Effects. World Scientific, New York, 2010. 
[19] Roland R Netz and Dominik Horinek. Progress in modeling of ion effects at the vapor/water interface. Annual review of physical chemistry, 63:401-418, 2012.

[20] *Philippe Hünenberger and Maria Rief. Single-Ion Solvation: Experimental and Theoretical Approaches to Elusive Thermodynamic Quantities. RSC Publishing, Cambridge, 2011.

[21] *Lukas Vlcek, Ariel A Chialvo, and J Michael Simonson. Correspondence between cluster-ion and bulk solution thermodynamic properties: on the validity of the cluster-pair-based approximation. The Journal of Physical Chemistry A, 117(44):11328-11338, 2013.

[22] *Travis Pollard and Thomas L Beck. Quasichemical analysis of the cluster-pair approximation for the thermodynamics of proton hydration. The Journal of chemical physics, 140(22):224507-1, 2014.

[23] ${ }^{* *}$ Travis P Pollard and Thomas L Beck. The thermodynamics of proton hydration and the electrochemical surface potential of water. The Journal of chemical physics, 141(18):18C512-1, 2014. Derives new values for the fundamental thermodynamic quantities for proton hydration in a way that is free of extrathermodynamic assumptions and establishes a new value for the net potential of the water surface.

[24] ** Lukas Vlcek and Ariel A Chialvo. Single-ion hydration thermodynamics from clusters to bulk solutions: Recent insights from molecular modeling. Fluid Phase Equilibria, 407:58-75, 2016. Gives an overview of the history and current status of studies of single-ion hydration.

[25] BE Conway. The evaluation and use of properties of individual ions in solution. Journal of Solution Chemistry, 7(10):721-770, 1978.

[26] Thomas L Beck. The influence of water interfacial potentials on ion hydration in bulk water and near interfaces. Chemical Physics Letters, 561:1-13, 2013.

[27] *John C Conboy and Geraldine L Richmond. Examination of the electrochemical interface between two immiscible electrolyte solutions by second harmonic generation. The Journal of Physical Chemistry B, 101(6):983-990, 1997.

[28] James K Beattie, Alex M Djerdjev, and Gregory G Warr. The surface of neat water is basic. Faraday discussions, 141:31-39, 2009. 
[29] Himanshu Mishra, Shinichi Enami, Robert J Nielsen, Logan A Stewart, Michael R Hoffmann, William A Goddard, and Agustín J Colussi. Brønsted basicity of the air-water interface. Proceedings of the National Academy of Sciences, 109(46):18679-18683, 2012.

[30] *John L Fulton, Gregory K Schenter, Marcel D Baer, Christopher J Mundy, Liem X Dang, and Mahalingam Balasubramanian. Probing the hydration structure of polarizable halides: a multiedge XAFS and molecular dynamics study of the iodide anion. The Journal of Physical Chemistry B, 114(40):12926-12937, 2010 .

[31] Ming Hsin Cheng, Karen M Callahan, Alexandria M Margarella, Douglas J Tobias, John C Hemminger, Hendrik Bluhm, and Maria J Krisch. Ambient pressure X-ray photoelectron spectroscopy and molecular dynamics simulation studies of liquid/vapor interfaces of aqueous $\mathrm{NaCl}, \mathrm{RbCl}$, and $\mathrm{RbBr}$ solutions. The Journal of Physical Chemistry C, 116(7):4545-4555, 2012.

[32] Dale E Otten, Patrick R Shaffer, Phillip L Geissler, and Richard J Saykally. Elucidating the mechanism of selective ion adsorption to the liquid water surface. Proceedings of the National Academy of Sciences, 109(3):701-705, 2012.

[33] Susanne Dengler, Angelika Klaus, Gordon JT Tiddy, and Werner Kunz. How specific are ion specificities? a pilot NMR study. Faraday discussions, 160:121$133,2013$.

[34] Pavel Jungwirth and Douglas J. Tobias. Specific ion effects at the air/water interface. Chemical Reviews, 106(4):1259-1281, 2006.

[35] *Marielle Soniat, Grayson Pool, Lisette Franklin, and Steven W Rick. Ion association in aqueous solution. Fluid Phase Equilibria, 407:31-38, 2015.

[36] Yi Yao, Yosuke Kanai, and Max L Berkowitz. Role of charge transfer in water diffusivity in aqueous ionic solutions. The Journal of Physical Chemistry Letters, 5(15):2711-2716, 2014.

[37] *D Asthagiri, Lawrence R Pratt, Michael E Paulaitis, and Susan B Rempe. Hydration structure and free energy of biomolecularly specific aqueous dications, including $\mathrm{Zn}^{2+}$ and first transition row metals. Journal of the American Chemical Society, 126(4):1285-1289, 2004. 
[38] Thomas L. Beck, Michael E. Paulaitis, and Lawrence R. Pratt. The Potential Distribution Theorem and Models of Molecular Solutions. Cambridge, Cambridge, 2006.

[39] *D. Sabo, D. Jiao, S. Varma, L. R. Pratt, and S. B. Rempe. Case study of $\mathrm{Rb}^{+}(\mathrm{aq})$, quasi-chemical theory of ion hydration, and the no split occupancies rule. Annu. Rep. Prog. Chem., Sect. C: Phys. Chem., 109:266-278, 2013.

[40] Thomas L Beck. Hydration free energies by energetic partitioning of the potential distribution theorem. Journal of Statistical Physics, 145(2):335-354, 2011.

[41] Ayse Arslanargin and Thomas L. Beck. Free energy partitioning analysis of the driving forces that determine ion density profiles near the water liquid-vapor interface. The Journal of Chemical Physics, 136(10):104503-1, 2012.

[42] *Thomas L Beck. A local entropic signature of specific ion hydration. The Journal of Physical Chemistry B, 115(32):9776-9781, 2011.

[43] A. J. Stone. The Theory of Intermolecular Forces. Clarendon Press, Oxford, 1996.

[44] Konrad Patkowski, Bogumil Jeziorski, and Krzysztof Szalewicz. Unified treatment of chemical and van der waals forces via symmetry-adapted perturbation expansion. The Journal of Chemical Physics, 120(15):6849-6862, 2004.

[45] Anthony J Stone and Alston J Misquitta. Charge-transfer in symmetry-adapted perturbation theory. Chemical Physics Letters, 473(1):201-205, 2009.

[46] R. F. W. Bader. Atoms in Molecules: a Quantum Theory. Clarendon Press, Oxford, 1994.

[47] Enrico Ronca, Leonardo Belpassi, and Francesco Tarantelli. A quantitative view of charge transfer in the hydrogen bond: The water dimer case. ChemPhysChem, 15(13):2682-2687, 2014.

[48] Christopher J Fennell, Alan Bizjak, Vojko Vlachy, and Ken A Dill. Ion pairing in molecular simulations of aqueous alkali halide solutions. The Journal of Physical Chemistry B, 113(19):6782-6791, 2009. Correction 113, 14837-14838.

[49] *Yun Luo, Wei Jiang, Haibo Yu, Alexander D MacKerell, and Benoît Roux. Simulation study of ion pairing in concentrated aqueous salt solutions with a polarizable force field. Faraday discussions, 160:135-149, 2013. 
[50] *Eva Pluharová, Marcel D Baer, Gregory K Schenter, Pavel Jungwirth, and Christopher J Mundy. Dependence of the rate of LiF ion-pairing on the description of molecular interaction. The Journal of Physical Chemistry B, 2015. DOI: 10.1021/acs.jpcb.5b09344.

[51] J. W. Gibbs. The Collected Works of J. Willard Gibbs. Longmans, Green, and Co., New York, 1928. p. 429.

[52] E. A. Guggenheim. The conceptions of electrical potential difference between two phases and the individual activities of ions. J. Phys. Chem., 33:842-849, 1928.

[53] E. A. Guggenheim. Thermodynamics: An Advanced Treatment for Chemists and Physicists. North Holland, New York, 1957. p. 374.

[54] ** Lawrence R Pratt. Contact potentials of solution interfaces: phase equilibrium and interfacial electric fields. The Journal of Physical Chemistry, 96(1):25-33, 1992. Seminal paper establishing fundamental aspects of interfacial potentials.

[55] W. Ronald Fawcett. Liquids, Solutions, and Interfaces: From Classical Macroscopic Descriptions to Modern Microscopic Details. Oxford UniversityOxford, Oxford, 2004.

[56] Kevin Leung. Surface potential at the air- water interface computed using density functional theory. The Journal of Physical Chemistry Letters, 1(2):496499, 2009.

[57] Shawn M Kathmann, I-Feng William Kuo, Christopher J Mundy, and Gregory K Schenter. Understanding the surface potential of water. The Journal of Physical Chemistry B, 115(15):4369-4377, 2011.

[58] Edward Harder and Benoît Roux. On the origin of the electrostatic potential difference at a liquid-vacuum interface. The Journal of chemical physics, 129(23):234706-1, 2008.

[59] R. C. Remsing, M. D. Baer, G. K. Schenter, C. J. Mundy, and J. D. Weeks. The role of broken symmetry in solvation of a spherical cavity in classical and quantum water models. The Journal of Physical Chemistry Letters, 5(16):2767$2774,2014$. 
[60] G Lee Warren and Sandeep Patel. Hydration free energies of monovalent ions in transferable intermolecular potential four point fluctuating charge water: An assessment of simulation methodology and force field performance and transferability. The Journal of chemical physics, 127(6):064509-1, 2007.

[61] * Yu Shi and Thomas L Beck. Length scales and interfacial potentials in ion hydration. The Journal of chemical physics, 139(4):044504-1, 2013.

[62] William A Donald and Evan R Williams. An improved cluster pair correlation method for obtaining the absolute proton hydration energy and enthalpy evaluated with an expanded data set. The Journal of Physical Chemistry B, 114(41):13189-13200, 2010.

[63] Michael D Tissandier, Kenneth A Cowen, Wan Yong Feng, Ellen Gundlach, Michael H Cohen, Alan D Earhart, James V Coe, and Thomas R Tuttle. The proton's absolute aqueous enthalpy and gibbs free energy of solvation from cluster-ion solvation data. The Journal of Physical Chemistry A, 102(40):77877794, 1998.

[64] Dor Ben-Amotz and Robin Underwood. Unraveling water's entropic mysteries: a unified view of nonpolar, polar, and ionic hydration. Accounts of chemical research, 41(8):957-967, 2008.

[65] *Lorand Horváth, Titus Beu, Manoel Manghi, and John Palmeri. The vaporliquid interface potential of (multi) polar fluids and its influence on ion solvation. The Journal of chemical physics, 138(15):154702-1, 2013.

[66] Joseph R Cendagorta and Toshiko Ichiye. The surface potential of the watervapor interface from classical simulations. The Journal of Physical Chemistry $B, 119: 9114-9122,2015$.

[67] *D Asthagiri, Lawrence R Pratt, and HS Ashbaugh. Absolute hydration free energies of ions, ion-water clusters, and quasichemical theory. The Journal of chemical physics, 119(5):2702-2708, 2003.

[68] Henry S Ashbaugh and D Asthagiri. Single ion hydration free energies: A consistent comparison between experiment and classical molecular simulation. The Journal of chemical physics, 129(20):204501-1, 2008.

[69] Yan Levin, Alexandre P Dos Santos, and Alexandre Diehl. Ions at the airwater interface: an end to a hundred-year-old mystery? Physical review letters, 103(25):257802-1, 2009. 
[70] Tatsuya Ishiyama and Akihiro Morita. Molecular dynamics study of gas-liquid aqueous sodium halide interfaces. I. flexible and polarizable molecular modeling and interfacial properties. The Journal of Physical Chemistry C, 111(2):721-737, 2007.

[71] Daniel JVA dos Santos, Florian Muüller-Plathe, and Volker C Weiss. Consistency of ion adsorption and excess surface tension in molecular dynamics simulations of aqueous salt solutions. The Journal of Physical Chemistry C, 112(49):19431-19442, 2008.

[72] Alan Grossfield, Pengyu Ren, and Jay W Ponder. Ion solvation thermodynamics from simulation with a polarizable force field. Journal of the American Chemical Society, 125(50):15671-15682, 2003.

[73] Yanjie Zhang, Steven Furyk, David E Bergbreiter, and Paul S Cremer. Specific ion effects on the water solubility of macromolecules: PNIPAM and the Hofmeister series. Journal of the American Chemical Society, 127(41):1450514510, 2005.

[74] Andrea Salis, Francesca Cugia, Drew F Parsons, Barry W Ninham, and Maura Monduzzi. Hofmeister series reversal for lysozyme by change in $\mathrm{pH}$ and salt concentration: insights from electrophoretic mobility measurements. Physical Chemistry Chemical Physics, 14(13):4343-4346, 2012.

[75] Francesca Cugia, Maura Monduzzi, Barry W Ninham, and Andrea Salis. Interplay of ion specificity, $\mathrm{pH}$ and buffers: insights from electrophoretic mobility and $\mathrm{pH}$ measurements of lysozyme solutions. RSC Advances, 3(17):5882-5888, 2013.

[76] B. Hessa and N. F. A. van der Vegt. Cation specific binding with protein surface charges. Proc. Natl. Acad. USA, 106:13296-13300, 2009.

[77] Nadine Schwierz, Dominik Horinek, and Roland R Netz. Anionic and cationic Hofmeister effects on hydrophobic and hydrophilic surfaces. Langmuir, 29(8):2602-2614, 2013.

[78] Poul B Petersen and Richard J Saykally. On the nature of ions at the liquid water surface. Annu. Rev. Phys. Chem., 57:333-364, 2006.

[79] *Marcel D Baer, Van-Thai Pham, John L Fulton, Gregory K Schenter, Mahalingam Balasubramanian, and Christopher J Mundy. Is iodate a strongly 
hydrated cation? The Journal of Physical Chemistry Letters, 2(20):2650-2654, 2011.

[80] Carl Caleman, Jochen S. Hub, Paul J. van Maaren, and David van der Spoel. Atomistic simulation of ion solvation in water explains surface preference of halides. Proceedings of the National Academy of Sciences, 108(17):6838-6842, April 2011.

[81] Zhen Zhao, David M. Rogers, and Thomas L. Beck. Polarization and charge transfer in the hydration of chloride ions. The Journal of Chemical Physics, 132(1):014502-1, January 2010.

[82] *Marcel D Baer and Christopher J Mundy. Toward an understanding of the specific ion effect using density functional theory. The Journal of Physical Chemistry Letters, 2(9):1088-1093, 2011.

[83] D Asthagiri, LR Pratt, and JD Kress. Ab initio molecular dynamics and quasichemical study of $\mathrm{H}^{+}$(aq). Proceedings of the National Academy of Sciences of the United States of America, 102(19):6704-6708, 2005.

[84] D Asthagiri, PD Dixit, S Merchant, ME Paulaitis, LR Pratt, Susan B Rempe, and Sameer Varma. Ion selectivity from local configurations of ligands in solutions and ion channels. Chemical physics letters, 485(1):1-7, 2010.

[85] Nathalia F Carvalho and Josefredo R Pliego. Cluster-continuum quasichemical theory calculation of the lithium ion solvation in water, acetonitrile and dimethyl sulfoxide: an absolute single-ion solvation free energy scale. Physical Chemistry Chemical Physics, 17(40):26745-26755, 2015.

[86] Jocelyn M Rodgers and John D Weeks. Local molecular field theory for the treatment of electrostatics. Journal of Physics: Condensed Matter, 20(49):494206, 2008 .

[87] Ayse Arslanargin, August Powers, Thomas L Beck, and Steven W Rick. Models of ion solvation thermodynamics in ethylene carbonate and propylene carbonate. The Journal of Physical Chemistry B, 120:1497-1508, 2016.

[88] Jared D. Smith, Richard J. Saykally, and Phillip L. Geissler. The effects of dissolved halide anions on hydrogen bonding in liquid water. Journal of the American Chemical Society, 129(45):13847-13856, November 2007. 
[89] Safir Merchant and D Asthagiri. Thermodynamically dominant hydration structures of aqueous ions. The Journal of chemical physics, 130(19):195102-1, 2009.

[90] *Bernhard Sellner, Marat Valiev, and Shawn M Kathmann. Charge and electric field fluctuations in aqueous $\mathrm{NaCl}$ electrolytes. The Journal of Physical Chemistry B, 117(37):10869-10882, 2013.

[91] Johnny C. Wu, Jean-Philip Piquemal, Robin Chaudret, Peter Reinhardt, and Pengyu Ren. Polarizable molecular dynamics simulation of $\mathrm{Zn}(\mathrm{II})$ in water using the AMOEBA force field. Journal of Chemical Theory and Computation, 6(7):2059-2070, July 2010.

[92] Matteo Dal Peraro, Simone Raugei, Paolo Carloni, and Michael L. Klein. SoluteSolvent Charge Transfer in Aqueous Solution. ChemPhysChem, 6(9):1715-1718, 2005 .

[93] Mohammed Ahmed, Ajay K. Singh, Jahur A. Mondal, and Sisir K. Sarkar. Water in the hydration shell of halide ions has significantly reduced Fermi resonance and moderately enhanced Raman cross section in the $\mathrm{OH}$ stretch regions. The Journal of Physical Chemistry B, 117(33):9728-9733, 2013.

[94] B. Sellner and S. M. Kathmann. A matter of quantum voltages. J. Chem. Phys., 141(18):18C534-1, 2014.

[95] Brad A. Bauer, Timothy R. Lucas, Alisa Krishtal, Christian Van Alsenoy, and Sandeep Patel. Variation of ion polarizability from vacuum to hydration: Insights from Hirshfeld partitioning. The Journal of Physical Chemistry A, 114(34):8984-8992, 2010.

[96] Marielle Soniat and Steven W. Rick. The effects of charge transfer on the aqueous solvation of ions. The Journal of Chemical Physics, 137(4):044511-1, July 2012.

[97] Qiang Zhang, Ruiting Zhang, Ying Zhao, HuanHuan Li, Yi Qin Gao, and Wei Zhuang. Pairing preferences of the model mono-valence mono-atomic ions investigated by molecular simulation. The Journal of chemical physics, 140(18):184504, 2014. 


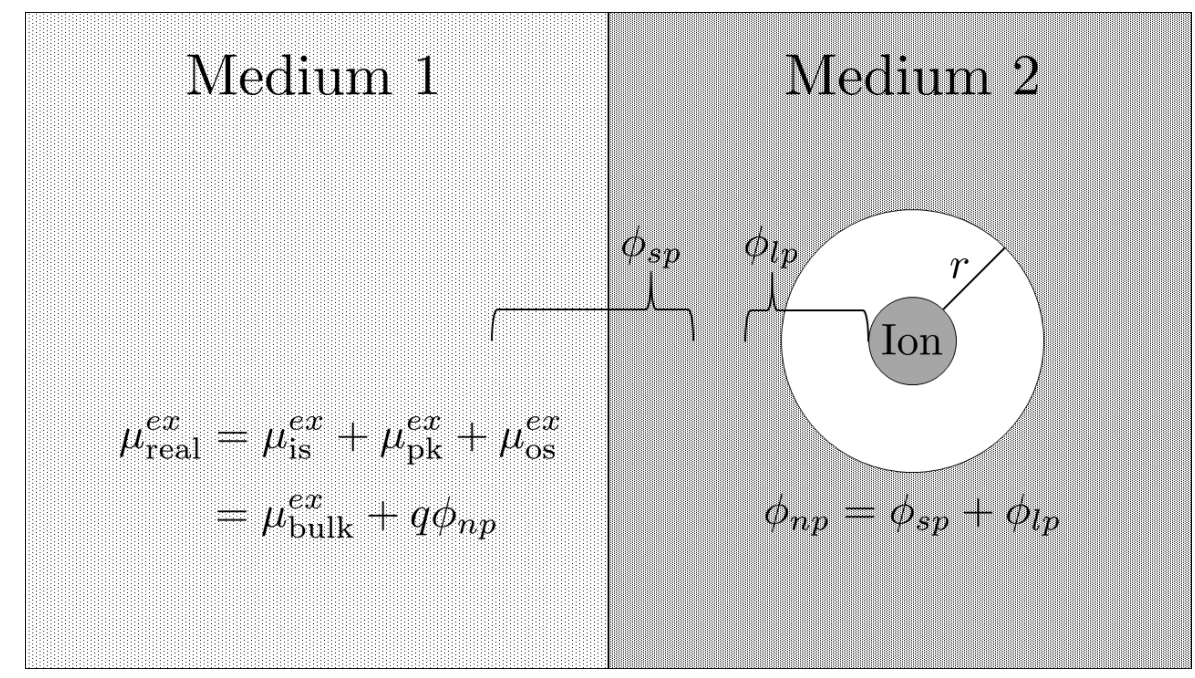

Figure 1: Schematic representation of quasichemical theory partitioning and interfacial potentials. For the discussion in the text Medium 1 is the vapor phase and Medium 2 is water. $\phi_{s p}$ is the potential shift crossing the vapor-liquid boundary, $\phi_{l p}$ is for the liquid-cavity boundary, and $\phi_{n p}$ is the sum of the two. Inside the liquid, a cavity is grown around the ion, with a radius $r$. The real free energy $\mu_{\text {real }}^{e x}$ is the work to move a single ion from the vapor into the liquid phase. It can be considered as a three-step process (as in quasichemical theory): 1) grow cavity, producing the packing term $\mu_{p}^{e x} 2$ ) compute interaction free energy for the ion in the cavity, the long-ranged term $\mu_{l r}^{e x}$ and 3) allow the solvent to relax into contact with the ion, the inner-shell term $\mu_{i s}^{e x}$. 


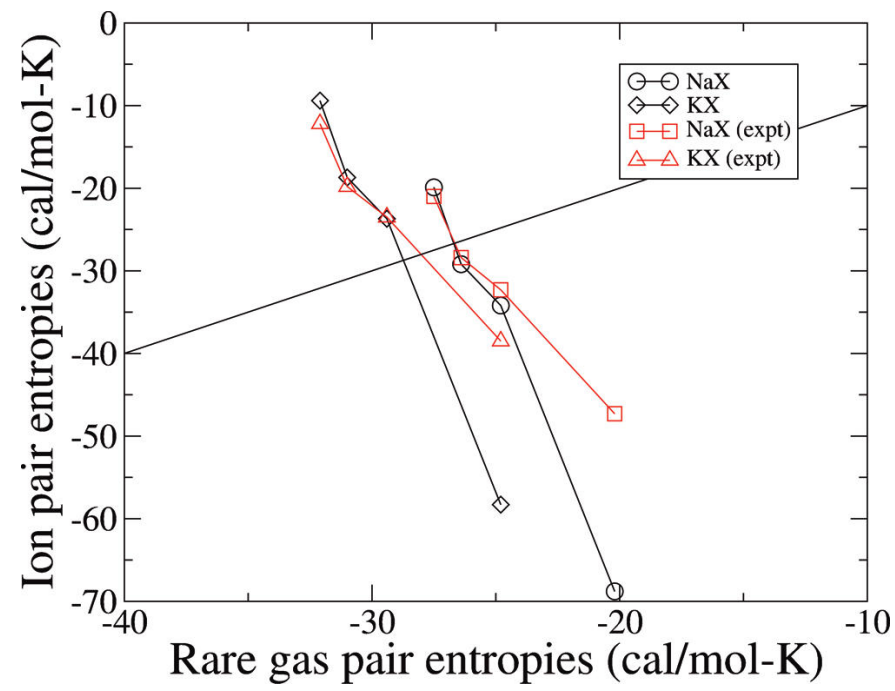

Figure 2: Shown are ion pair entropies vs. their rare gas pair counterparts. The KX sequence is on the left with $\mathrm{X}$ the halide $\left(\mathrm{F}^{-}, \mathrm{Cl}^{-}, \mathrm{Br}^{-}\right.$, and $\mathrm{I}^{-}$, with $\mathrm{F}^{-}$on the bottom). The corresponding NaX sequence is on the right. Experimental data are in red, and computed results are in black. Notice the significant error for the $\mathrm{F}^{-}$ion, which presents special challenges, see below. Further details can be found in Ref. [42]*. Reproduced from Ref. [42]* with permission. 


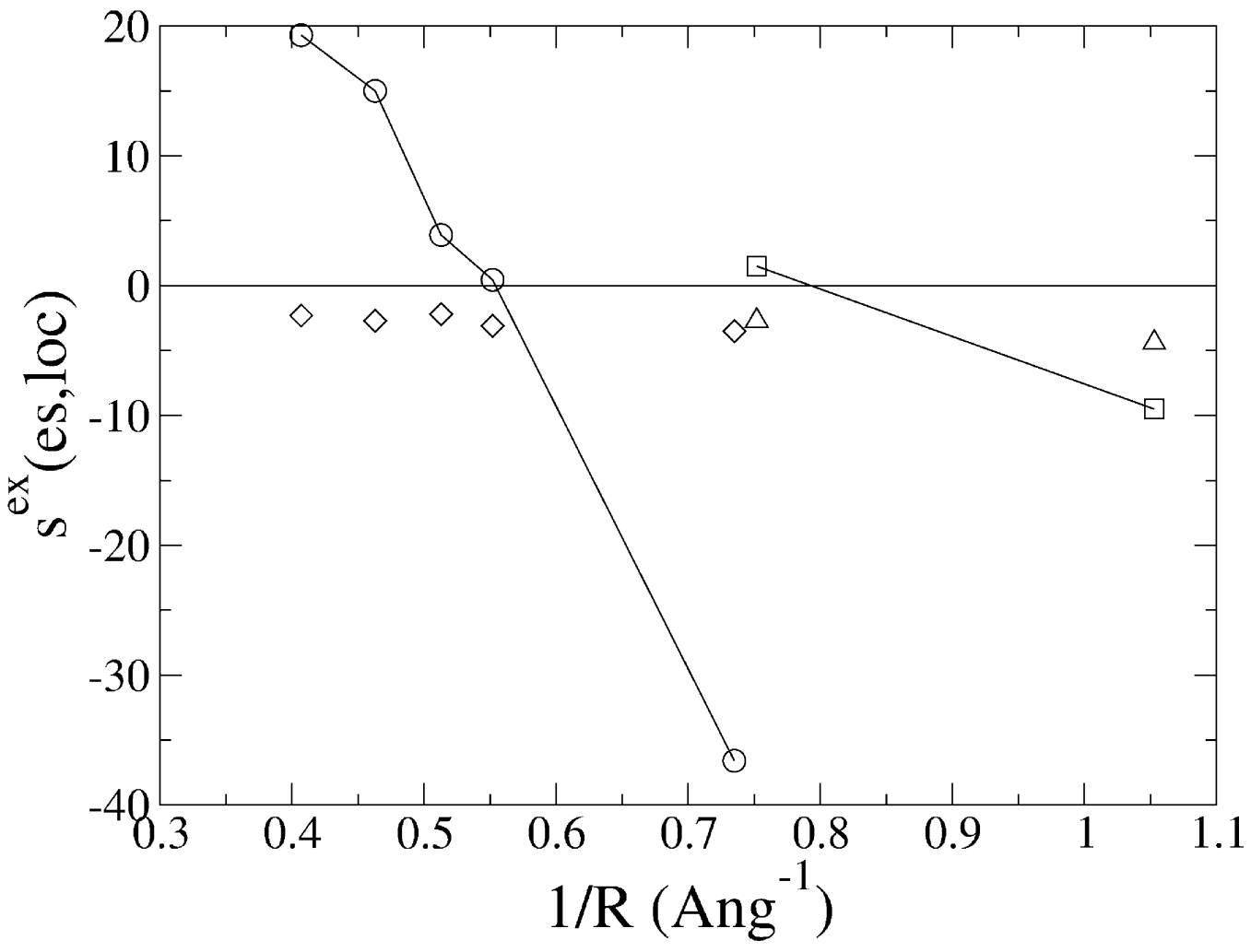

Figure 3: Figure showing local and far field electrostatic parts of the single-ion entropy as a function of the inverse of the ion crystal radius. The anion data is on the left $\left(\mathrm{F}^{-}\right.$starting at the bottom, $\mathrm{Cl}^{-}, \mathrm{Br}^{-}, \mathrm{I}^{-}$, and an artificially large $\mathrm{I}^{-}$ion). The cation data is on the right $\left(\mathrm{Na}^{+}\right.$and $\left.\mathrm{K}^{+}\right)$. The local contribution for the anions is labelled with an open circle, while the far-field contribution is labelled with an open diamond. The local contribution for the cations is labelled with an open square, while the far-field contribution is labelled with an open triangle. Note the strong ion specificity due to interactions near the ion and the small-magnitude and largely ion independent far-field contribution. Also note the very different slopes of the local contributions for anions vs. cations. Reproduced from Ref. [42]* with permission. 


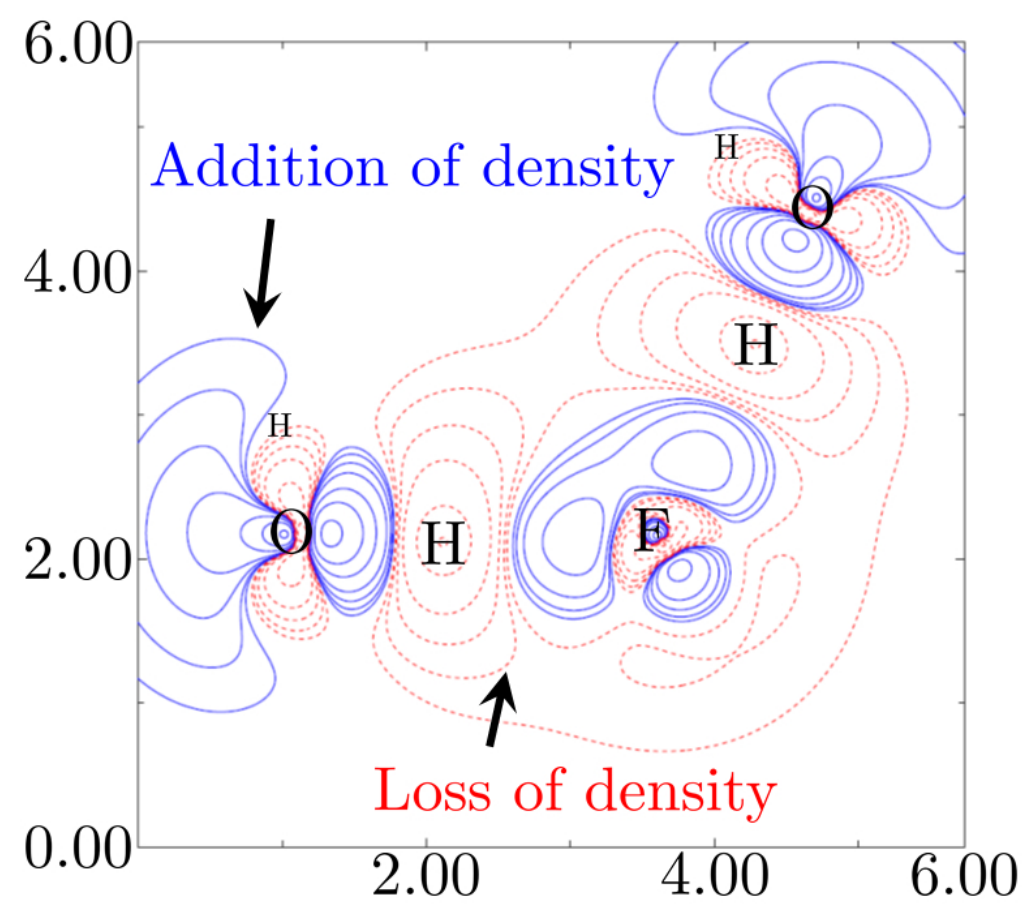

Figure 4: Shows charge density redistribution in the $\mathrm{F}^{-} / \mathrm{W}_{2}$ dimer cluster. The contours are obtained from the difference between the electronic charge density in the complex minus the individual ion and water charge densities. The complexity of the charge rearrangments is apparent. 


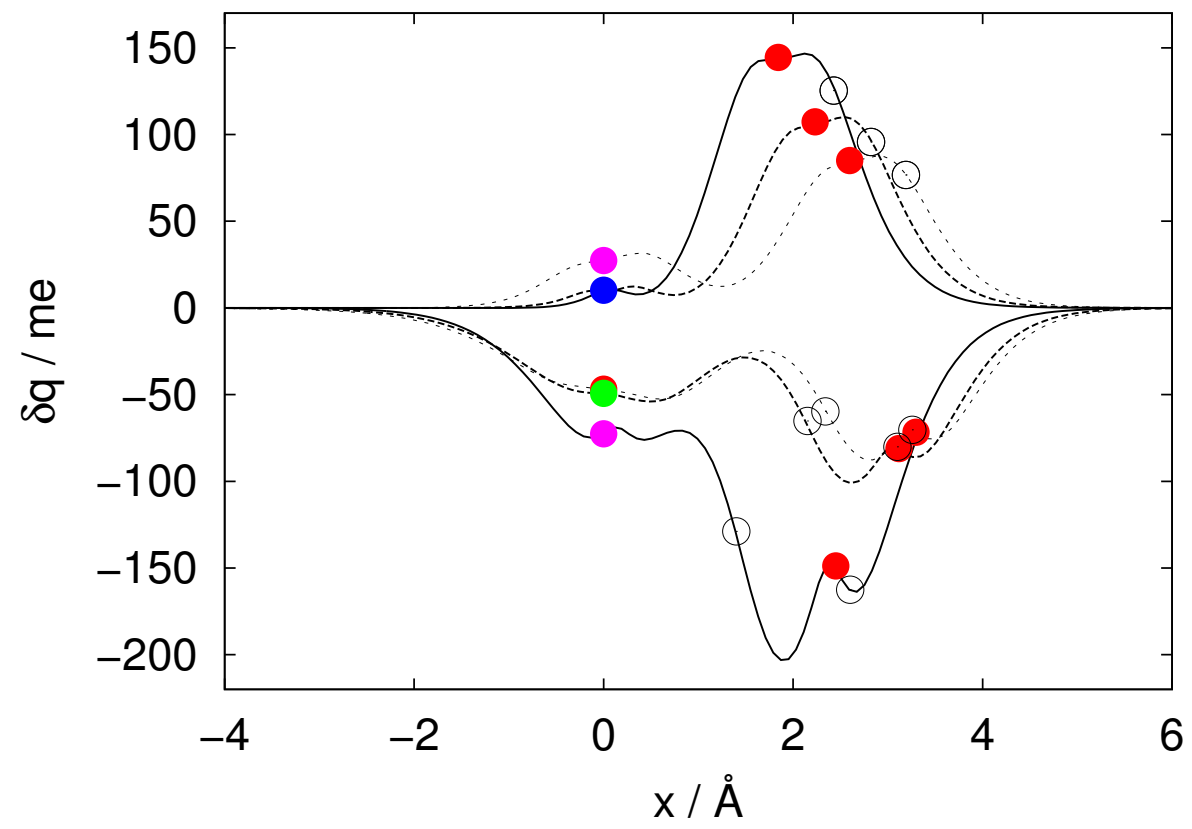

Figure 5: Charge displacement curves following Ref. [47]. The curves indicate both direction and magnitude of charge passing through a moving plane perpendicular to the ion-water bond and so offers a unique perspective of charge redistribution in the dimers. Ref. [47] and citations therein argue that an unambiguous estimation of the charge transferred can be evaluated at the minimum/maximum appearing in the curve between the ion and the nearest water atom positions. The integration proceeds from left to right and so a positive value like we obtain for cations (top curves) indicates charge flows from the right side to the left (water to ion) and vice versa for negative values like that obtained for anions (lower curves). All ions are located at $x=0$. Any displaced red and white cirlces signal oxygen and hydrogen positions, respectively. Ions are colored magenta $\left(\mathrm{F}^{-}\right)$, green $\left(\mathrm{Cl}^{-}\right)$, red $\left(\mathrm{Br}^{-}\right)$, larger red $\left(\mathrm{Li}^{+}\right)$, blue $\left(\mathrm{Na}^{+}\right)$, and magenta $\left(\mathrm{K}^{+}\right.$on top of the plot $)$. 
Addition of density Loss of density

$\left[\mathrm{F}\left(\mathrm{H}_{2} \mathrm{O}\right)_{3}\right]^{-}$

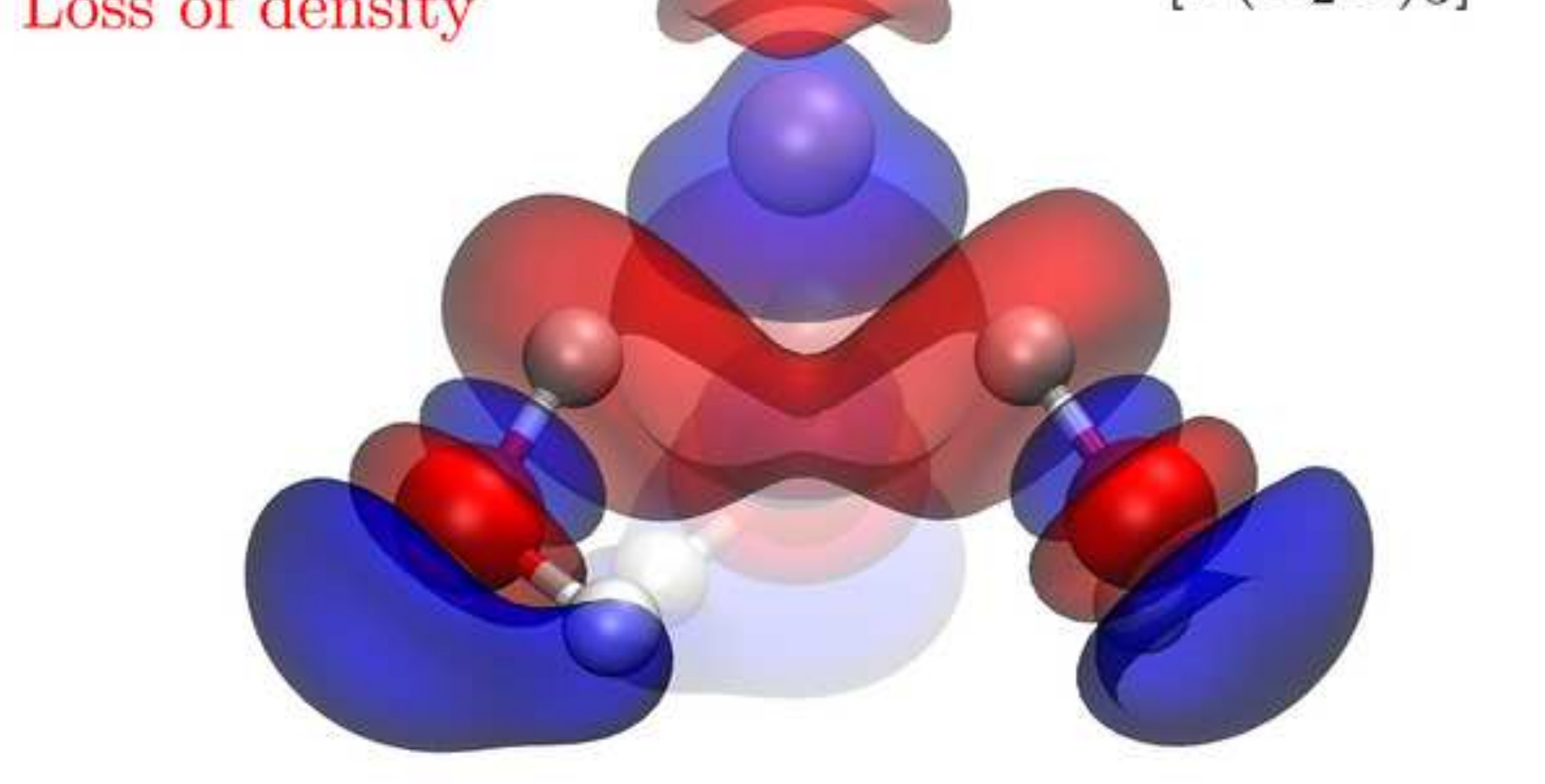

